\title{
Mujeres marginadas: Los desafíos impuestos por el género, la carencia de hogar y el VIH en la ciudad de Nueva York
}

\author{
Marginalized Women: Challenges of Gender, \\ Homelessness, and HIV in New York City
}

\begin{abstract}
Adele Weiner And Kim Lorber
Adele Weiner es doctora ylicenciada en Trabajo Social Clinico, actualmente es profesora en Audrey Cohen College of Human Services del Metropolitan College de Nueva York. Su dirección postal es 431 Canal Street, New York, NY 10013; su e-mail es aweiner@mcny.edu. Kim Lorber es Doctora y Licenciada en Trabajo Social Clínico, y es profesora en la Facultad de Ciencias Sociales y Servicios Humanos de Ramapo College de Nueva Jersey en Mahwah, Nueva Jersey. Su dirección postal es 505 Ramapo Valley Road • Mahwah, NJ 07430; su e-mail es klorber@ramapo.edu.
\end{abstract}

\begin{abstract}
Resumen
La vida de las mujeres sin hogar representa numerosos desafios y una compleja matriz que muestra inequidad de género, comercio sexual, uso de drogas y otros factores socioculturales, ubicándolas en riesgo de contraer VIH/Sida y exponiéndolas a la violencia, y a la muerte. Este estudio examina una muestra de hombres y mujeres urbanos marginados para determinar la forma en que el estatus de sin hogar y el género se relacionan con variables socioeconómicas.
\end{abstract}

Palabras clave (mujeres, sin hogar; Sida/HIV, género, pobreza)

\begin{abstract}
Life for homeless women presents numerous challenges and represents a complex matrix of gender inequality, sex trading, drug use and other socio-cultural factors, placing them at risk for HIV/AIDS, violence, and death. This study looks at a sample of marginalized urban women and men to determine how homeless status and gender are related to socioeconomic variables.
\end{abstract}

Key words (women, homelessness; HIV/AIDS, gender, poverty)

\section{Introducción}

Este estudio examina una muestra de hombres y mujeres urbanos marginados para determinar la forma en que la situación de calle y el género se relacionan con variables socioeconómicas. La muestra incluyó hombres y mujeres que recibieron asesoría y exámenes de VIH mediante un programa de ayuda callejera. Previo a explicar la metodología aplicada, se presenta el conocimiento acumulado respecto a la población que se estudia.

Las mujeres sin hogar se han identificado como población vulnerable, en riesgo de contraer enfermedades, expuesta a la violencia y al uso de drogas. Históricamente, las mujeres de bajos ingresos que usan drogas se han considerado como vectores de enfermedades médicas y morales (Mulia, 2000), cuando lo verdadero es probablemente a la inversa. Muchas de ellas ven insatisfechas sus necesidades médicas (Lewis, Andersen, \& Gelberg, 2003). Las mujeres solteras y sin hogar pueden haber experimentado eventos más estresantes que los hombres solteros sin hogar o que las mujeres con hijos sin hogar y es más probable que hayan experimentado altos niveles de abusos en la niñez, abusos físicos y sexuales (Zugazaga, 2004) los que pueden relacionarse con el abuso de sustancias y con enfermedades mentales (Christensen, Hodgkins, Garces, Estlund, Miller, \& Touchton, 2005). 
La mortalidad en los individuos sin hogar se estima tres veces mayor que la correspondiente a la población general y es probable que las mujeres sin hogar que han muerto hayan utilizado drogas inyectables (Barrow, Herman, Cordova, \& Streuning, 1999). Cheung y Hwang (2004) indicaron que "... el ser una mujer soltera sin hogar y tener menos de 45 años de edad se asocia con un riesgo de muerte agudizado..." (p. 1247). Este estudio encontró que, entre las mujeres sin hogar, la edad promedio de muerte era de 39 años y para aquellas mujeres de edades entre 18 y 44 años las principales causas de muerte fueron el VIH/SIDA y el abuso de substancias.

Las mujeres que usan drogas inyectables tienen niveles altos de conductas sexuales riesgosas, incluyendo el tener relaciones sexuales con tres o más parejas, el intercambio de sexo por dinero y/o por drogas, y tener una pareja que utiliza drogas inyectables (Harvey, Bird, De Rosa, Montgomery, \& Rohrbach, 2003). Las leyes y políticas relacionadas con el uso de drogas y el trabajo sexual, al igual que la ejecución de esas leyes por parte de la policía, afectan el contexto en el que se dan las conductas de riesgo (Blakenship \& Koester, 2002). El aumento en el uso del crack, fácilmente asequible, ha tenido un efecto negativo sobre las mujeres pobres que se involucran en el comercio sexual a cambio de dinero o drogas (Erickson, Butters, McGillicuddy, \& Hallgren, 2000). Las mujeres implicadas en el comercio sexual viven en condiciones de pobreza extrema, no tienen hogar, utilizan múltiples drogas y alcohol, han tenido experiencias traumáticas en su infancia (Weiner, 1996; Witte, El-Bassel, Wada, Gray, \& Wallace, 1999), y por lo común se ven expuestas al maltrato violento (Surrat \& Inciardi, 2004).

\section{Diseño metodológico}

Este estudio utiliza una muestra grande (siendo $\mathrm{N}=7749$ ) de hombres y mujeres marginados que recibieron asesoría y exámenes de VIH mediante un programa de ayuda callejera. A muchos de los individuos se les realizaron exámenes más de una vez, este estudio consideró específicamente su último o único examen. La información original incluyó 10.080 pruebas individuales; en este estudio la muestra se redujo a 6.737 para incluir a aquellos individuos a los que se realizó una única prueba, y los resultados de pruebas más recientes de 1.057 individuos. Una vez que un individuo arroja un resultado positivo en el examen del VIH no se le vuelve a aplicar el examen, aunque pueden seguir apareciendo en esta base de datos debido que se les procuran otros servicios. El estatus de VIH de esos individuos se mantiene positivo y la última visita para obtener servicios refleja la información más reciente proporcionada por el sujeto.

Los datos se recopilaron desde 1989 hasta marzo de 2004 mediante una serie de camionetas de ayuda que ofrecieron exámenes de VIH, educación sobre prevención, intercambio de agujas y gestión de los casos en toda la ciudad de Nueva York. Los equipos móviles le ofrecieron servicios a individuos en la calle y en las afueras de los albergues para personas sin hogar, centros sociales, cárceles, instalaciones de viviendas colectivas (SRO's) y bares. La información fue recolectada por el personal como parte de su entrega regular de insumos y servicios y fue ingresada en un sistema uniforme de informes solicitado por fuentes de financiamiento público. Los datos se exportaron para su análisis en el Paquete Estadístico de Ciencias Sociales (PECS).

El análisis de la información exploró las relaciones del género respecto del VIH, la raza, la etnia hispana, el trabajo sexual, el uso de drogas intravenosas y crack/cocaína por parte de los encuestados, el uso de drogas intravenosas y crack/cocaína por parte de sus parejas y/o su estatus respecto del VIH, y sus antecedentes de abuso sexual, violencia intrafamiliar o intentos suicidas. Una segunda serie de análisis estudió la relación entre la carencia de hogar y el género utilizando las mismas variables. Si bien muchos de los individuos fueron vistos más de una vez por los equipos móviles, este estudio se centra específicamente en sus visitas más recientes al servicio.

Las mujeres comprendieron $76,0 \%$ de los receptores del servicio; $22,5 \%$ fueron hombres y $1,5 \%$ fueron transexuales. Más de una quinta parte $(21,8 \%)$ de la muestra resultó VIH positiva y $10 \%$ no tenía hogar. El género se relacionó con el estatus de VIH $(p<, 05)$. Los individuos transexuales tuvieron la mayor probabilidad de resultar VIH positivos (50\%), seguidos de las mujeres $(23,8 \%)$ y por los hombres $(13,1 \%)$. Los hombres tuvieron mayor probabilidad de no tener hogar $(12,1 \%)$ en comparación con el porcentaje de $10,5 \%$ de las mujeres $(\mathrm{p}=, 049)$. La raza y la etnia se combinaron en este sistema público de informes. La mayor parte de la muestra fue afro-americana $(57,3 \%)$, $20,9 \%$ fue de color blanco, $21.2 \%$ era hispana y menos de $1 \%$ resultó provenir del Asia/Pacífico o ser de origen norteamericano. La Figura 1 indica el desglose de la muestra por género y por raza/etnia. Las mujeres comprenden la gran mayoría en todos los grupos étnicos. La disparidad entre mujeres y 
FIGURA 1

DESGLOSE DE LA MUESTRA SEGÚN GÉNERO Y RAZA/ETNIA

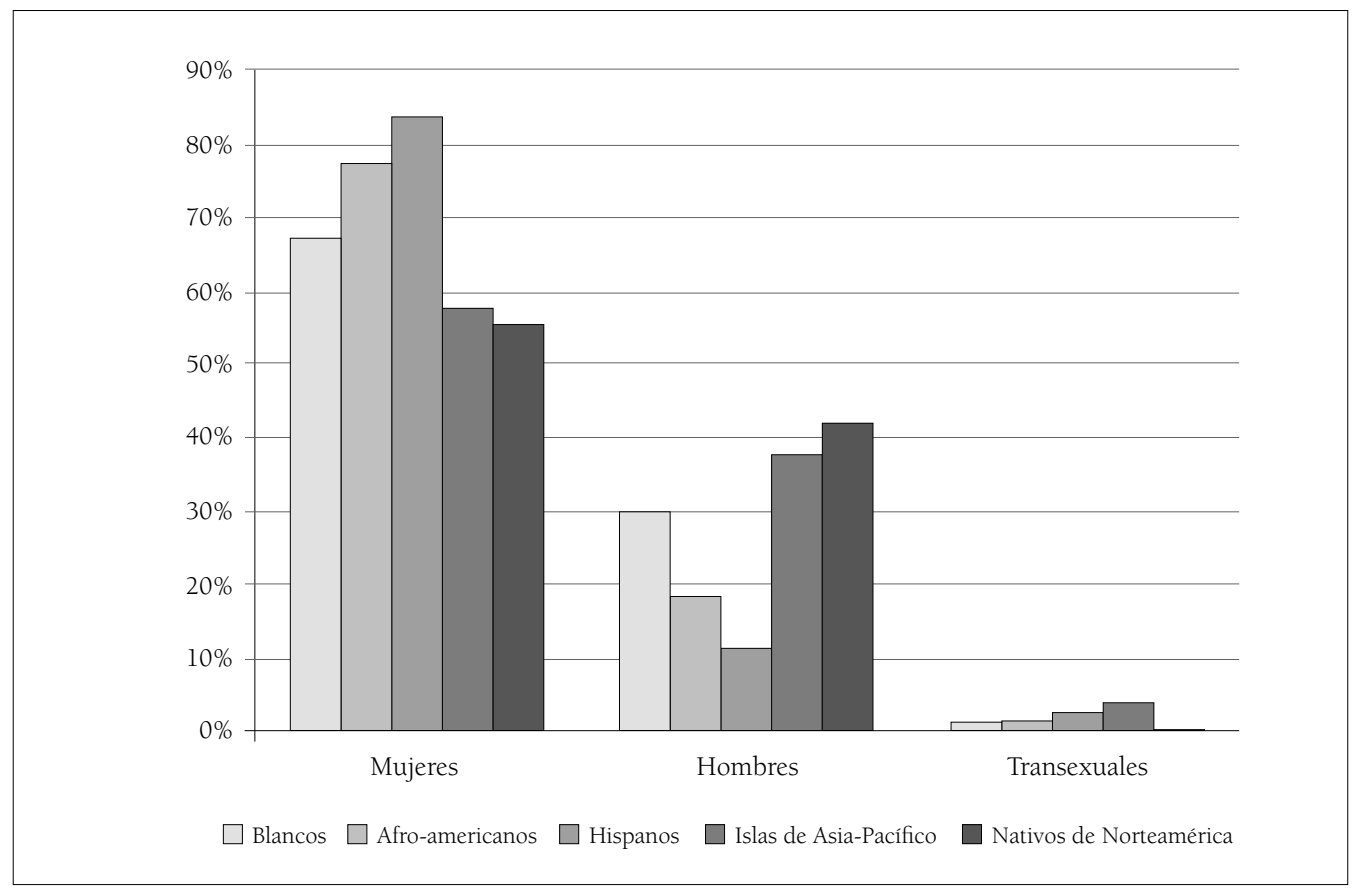

hombres es mayor entre los miembros hispanos de la muestra ( $84 \%$ mujeres y $13,1 \%$ varones). Al interior del segmento de los transexuales hay cantidades equivalentes de afro-americanos e hispanos ( $n=50$ ) pero éstos representan apenas 1,2\% de los afro-americanos y 3,0\% de los hispanos abarcados en la muestra. Sólo 16 individuos de raza blanca $(0,9 \%)$ se identificaron como transexuales. Si bien la cantidad total de personas provenientes de las Islas de Asia-Pacifico es pequeña en esta muestra
( $\mathrm{n}=45)$, los individuos transexuales comprenden $4,4 \%$ de este grupo.

Los antecedentes de trabajo sexual se informaron en $14,4 \%$ de la muestra del estudio, comprendiendo las mujeres la mayoría de los trabajadores sexuales $(85,8 \%)$. Entre los individuos transexuales se informó de 42,9\% con antecedentes de trabajo sexual en comparación con el $22 \%$ de todas las mujeres y el $4,5 \%$ de todos los hombres. Ver Figura 2. 
FIGURA 2

TRABAJO SEXUAL SEGÚN GÉNEROS

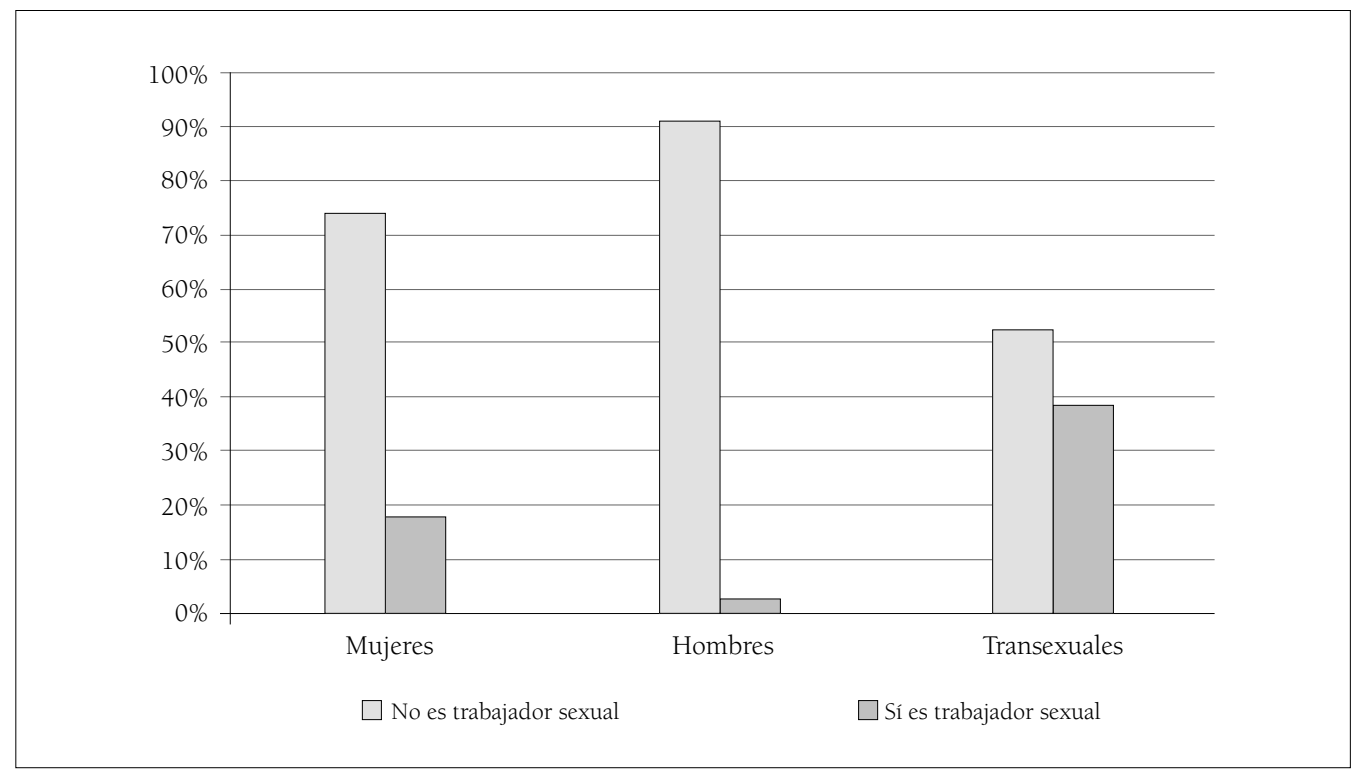

Esta población demostró niveles bastante elevados de uso de drogas. Más de una tercera parte de la muestra usaba crack $(34,8 \%)$ o cocaína $(38,2 \%)$, y $20 \%$ había utilizado drogas intravenosas. Muchos de ellos tenían una pareja con antecedentes de uso de drogas intravenosas (24\%) o era VIH positivo (17\%). Los individuos transexuales revelaron las tasas más elevadas de uso de cocaína $(57,8 \%)$ o tuvieron mayor probabilidad de tener parejas con antecedentes de uso de drogas (30,8\%). Ver Figura 3.

FIGURA 3

FACTORES DE RIESGO DE VIH SEGÚN GÉNEROS

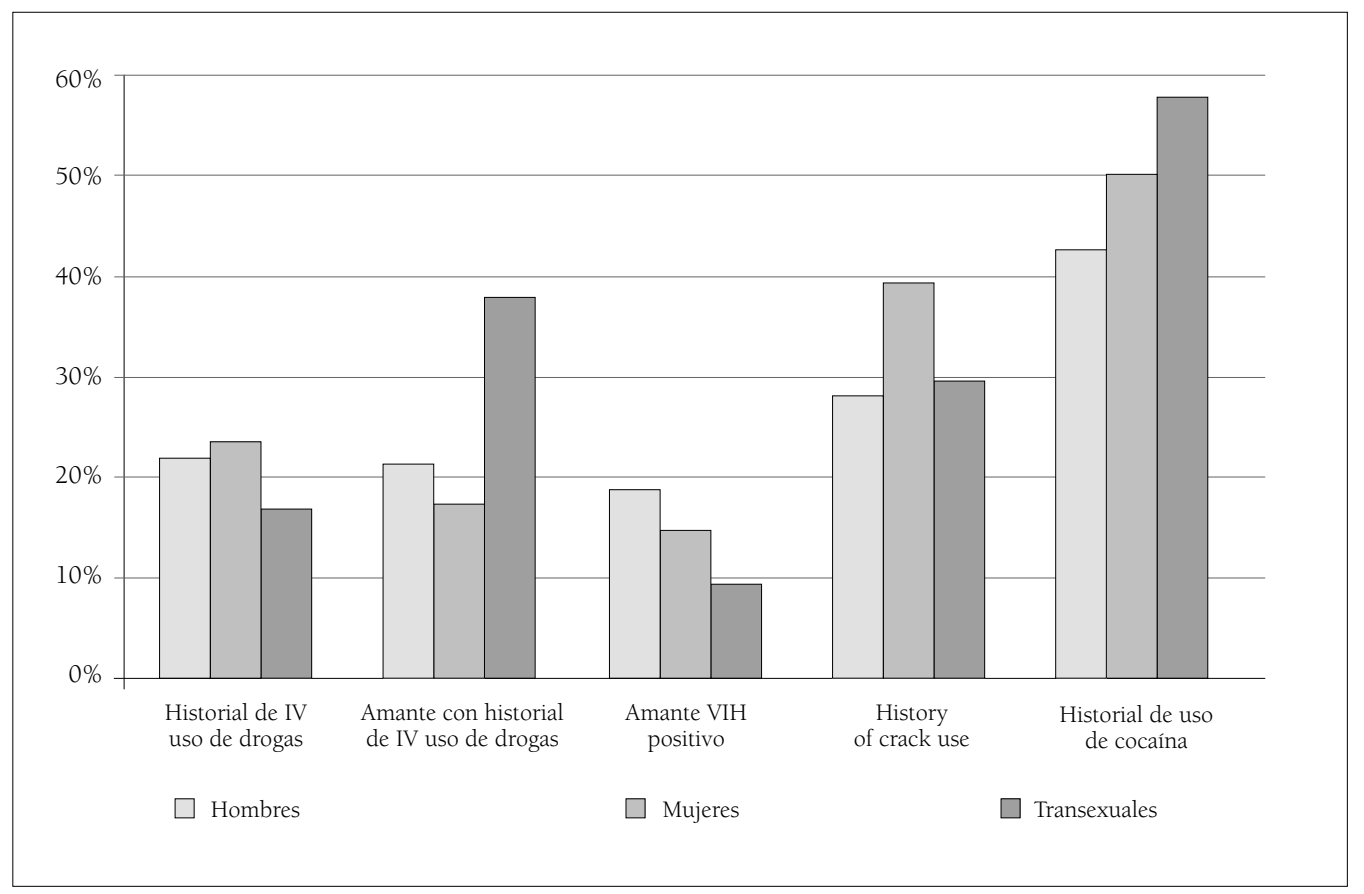


Sólo 7\% de las mujeres contaba con algún tipo de seguro de salud, y apenas dos individuos indicaron contar con cobertura de salud privada.

\section{Conclusiones con respecto a las diferencias de género}

La muestra incluyó a 5.923 mujeres (76\%), 1.750 hombres $(22,5 \%)$ y a 120 individuos transexuales (1,5\%). Originalmente, todos los análisis de datos en relación al género incluyeron a los individuos transexuales, los que parecieron representar la mayor parte de las conclusiones importantes en relación a género y constituyeron una parte relativamente pequeña de la muestra. Los individuos transexuales tuvieron menor probabilidad de carecer de hogar o de ser víctimas de violencia intrafamiliar y tuvieron mayor probabilidad de ser VIH positivos, de usar drogas inyectables, ser víctimas del abuso sexual y de ser trabajadores sexuales $(\mathrm{p}<, 05)$. Un porcentaje mucho mayor (50\%) de la población transexual resultó VIH positiva en comparación con las mujeres $(23,8 \%)$ o con los hombres $(13,1 \%, \mathrm{p}<, 05)$. Ver Figura 4.

El siguiente análisis de datos eliminó de la muestra a la pequeña cantidad de individuos transexuales ( $n=120)$ y sólo comparó hombres con mujeres. Se compararon diversos factores socio-culturales de hombres y mujeres, incluyendo estatus de VIH, uso de drogas intravenosas, crack y cocaína; tener una pareja VIH positivo o con antecedentes de uso de drogas intravenosas; haber experimentado abuso sexual o violencia intrafamiliar; haberse desempeñado como trabajador sexual; y carecer de hogar.

ESTATUS DE VIH SEGÚN GÉNERO

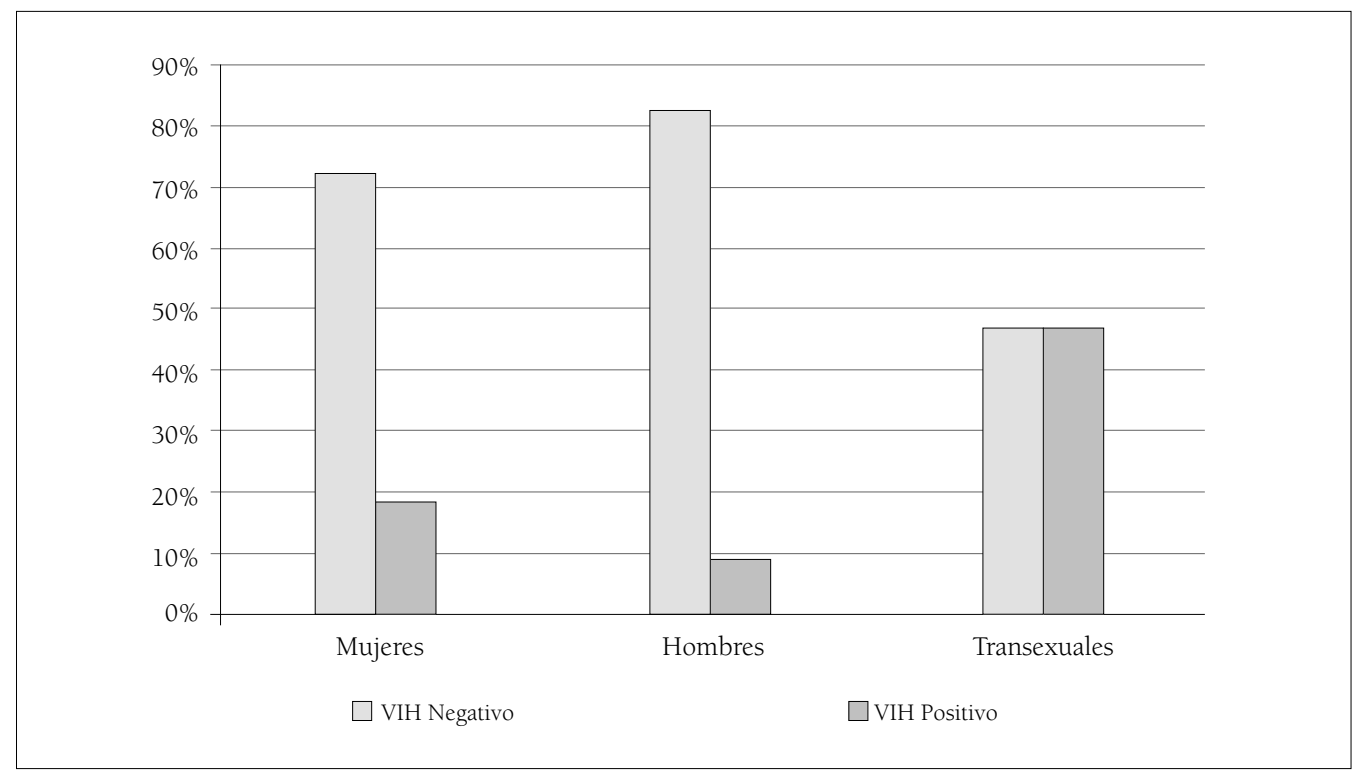


Se encontró una cantidad de diferencias de género y las mujeres marginadas parecieron estar más expuestas al contagio de VIH, uso de drogas y violencia. Una proporción mayor de las mujeres fue VIH positiva ( $<<, 05$, ver Figura 4), utilizó crack ( $p<, 05)$, tenía antecedentes de abuso sexual ( $\mathrm{p}<, 05)$, estuvo sometida a violencia intrafamiliar ( $p<, 05)$, tuvo intentos de suicidio $(\mathrm{p}=0.007)$, o se involucró en trabajo sexual $(\mathrm{p}<, 05)$. Ver Figura 5.

Los hombres tuvieron mayor probabilidad que las mujeres de involucrarse en alguna conducta de alto riesgo. Ellos mostraron tasas más elevadas de uso de cocaína ( $p=, 029)$. No se encontraron importantes diferencias de género entre hombres y mujeres con respecto a tener una pareja que usara drogas intravenosas, tener una pareja con VIH positivo, o sin hogar. Las conclusiones relativas al estatus de VIH o de uso de drogas intravenosas de sus parejas pueden relacionarse con la falta de conocimiento en la mujer. Esta población de mujeres mantenía múltiples relaciones de pareja y/o estuvo involucrada con hombres que no se mostraron proclives a compartir su estatus de VIH positivo o sus antecedentes de uso de drogas con sus parejas sexuales. Por temor a la violencia o al abandono, a menudo estas mujeres no hacen preguntas al respecto. Esto aumenta su exposición al contagio con VIH.

FIGURA 5

DIFERENCIAS IMPORTANTES ENTRE MUJERES Y HOMBRES

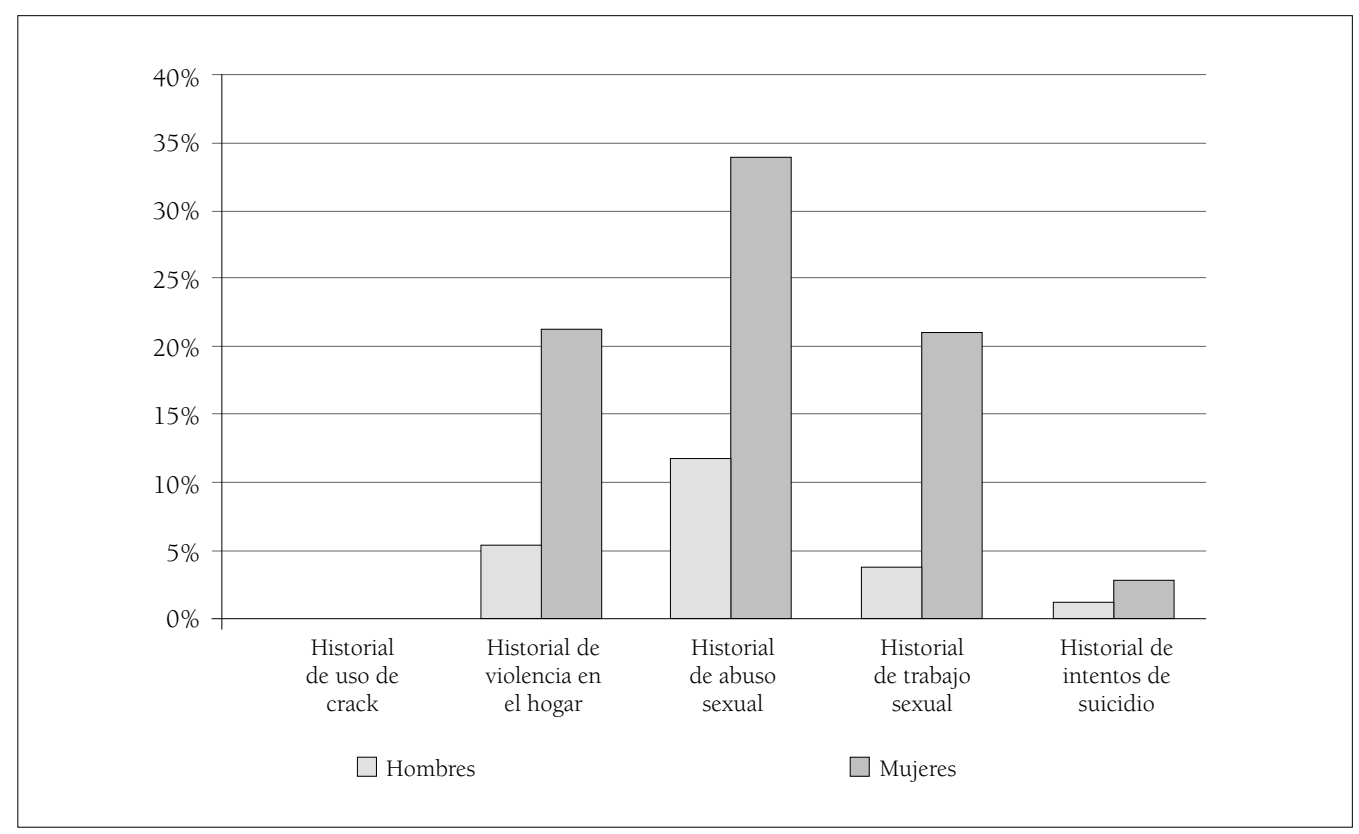




\section{Conclusiones relativas a las mujeres y al estatus de $\mathrm{VlH}$}

Una serie de análisis que usó el subconjunto de datos sobre mujeres $(n=5923)$ examinó las relaciones entre los factores socio-culturales y el estatus de VIH. Entre las mujeres con VIH el ser seropositivas se relacionó con diversos factores de riesgo identificados. Las mujeres involucradas en trabajo sexual tuvieron más probabilidad de ser VIH positivas $(\mathrm{p}=.001)$. Ver Figura 6, abajo.

Las mujeres con parejas que tenían antecedentes de uso de drogas intravenosas o VIH positivos tuvieron mayor probabilidad de ser VIH positivas $(\mathrm{p}<, 05)$. De las mujeres que tenían una pareja VIH positivo, $89,1 \%$ tenía relaciones sexuales con hombres. Debido a las vías de contagio del VIH, el mantener relaciones sexuales con hombres expone a las mujeres a un mayor riesgo de contraer la infección. La mayoría de las mujeres que participaron en esta muestra tuvieron relaciones sexuales con hombres que estaban contagiados con VIH o que tenían antecedentes de uso de drogas intravenosas. Las mujeres que tenían antecedentes personales de uso de drogas intravenosas o de crack mostraron tasas más elevadas de contagio con VIH $(\mathrm{p}<, 05)$. Ver Figura 7.

FIGURA 6

COMPARACIÓN DEL ESTATUS DE LAS TRABAJADORAS SEXUALES Y QUE NO LO SON

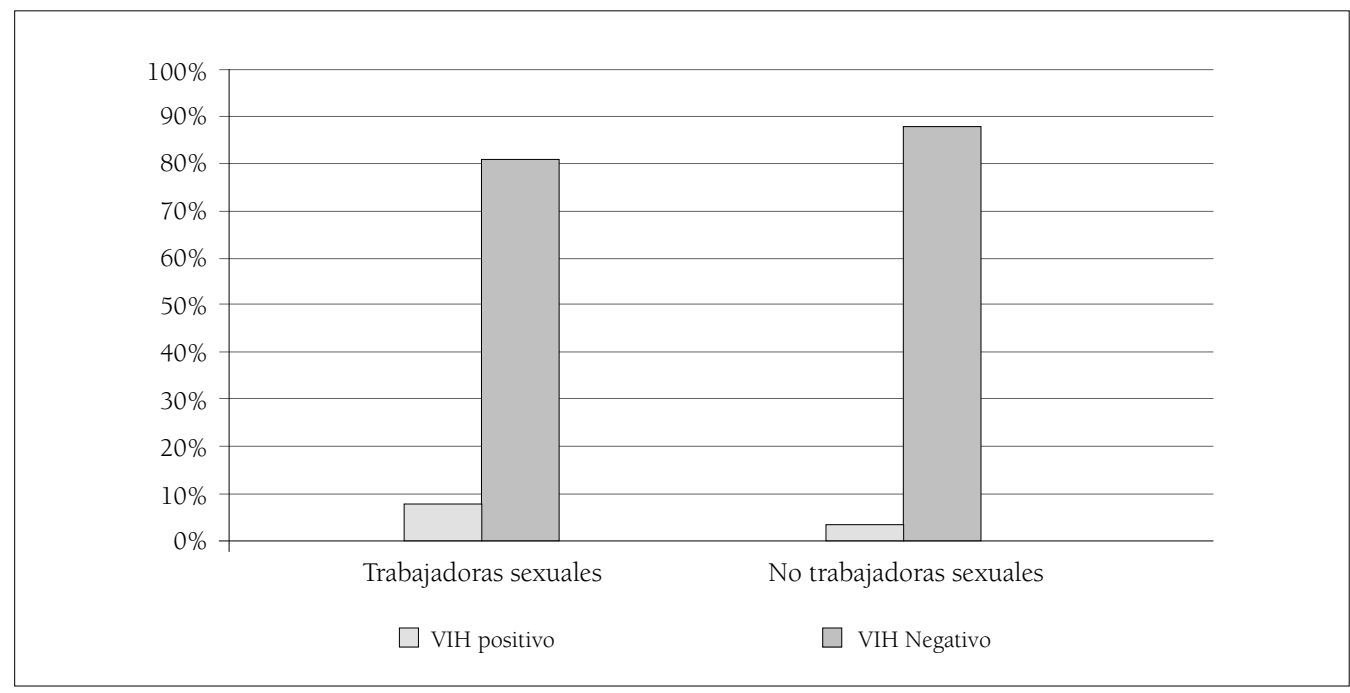

FIGURA 7

FACTORES IMPORTANTES DE RIESGO DE CONTAGIO CON VIH ENTRE LAS MUJERES

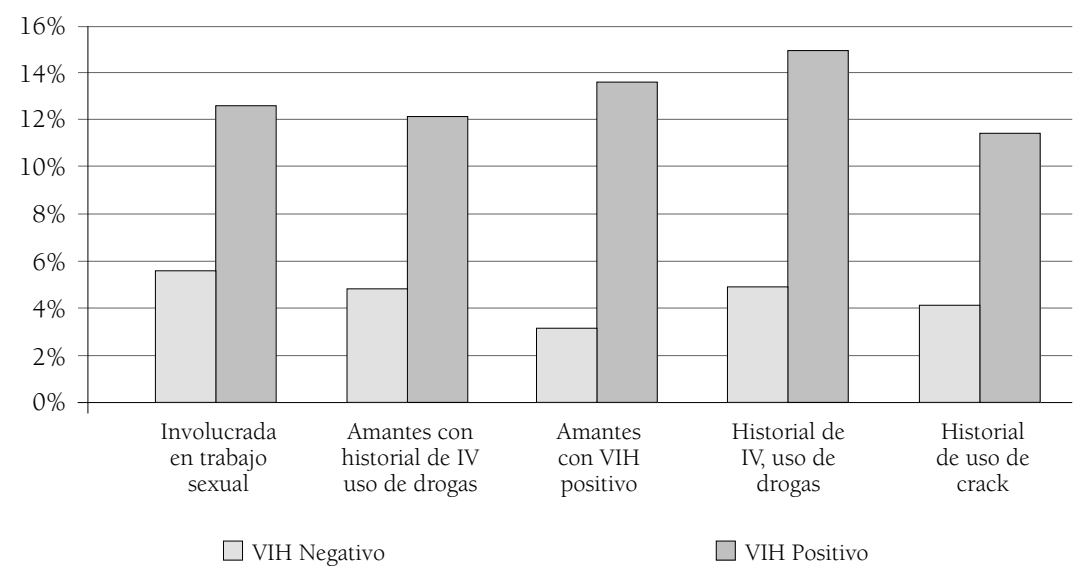




\section{Conclusiones sobre las mujeres y la carencia de hogar}

Se exploró también la relación entre la carencia de hogar y los factores de riesgo al interior de esta muestra de mujeres. Las mujeres con antecedentes de uso de drogas intravenosas tuvieron mayor posibilidad de contar con algún tipo de vivienda $(\mathrm{p}=, 004)$. Las mujeres con VIH positivo tuvieron también mayor probabilidad de contar con algún tipo de vivienda y no demostraron una condición de "sin hogar" ( $n=5347, p<, 05)$. Esto pudo deberse a una diversidad de factores, incluyendo el de la disponibilidad de hospedaje relacionada con su estatus de VIH positivo o de abuso de sustancias. Las mujeres más saludables demostraron menos opciones de vivienda. Ver Figura 8.

Entre las mujeres con antecedentes de abuso sexual parece darse un patrón inverso (Ver Figura 8). Las mujeres que informaron haber sido víctimas de abuso sexual tuvieron mayor probabilidad de estar sin hogar $(\mathrm{p}<, 05)$. Ciertamente, es posible y probable que esas mujeres hayan sido abusadas sexualmente debido a su carencia de hogar, lo que las hace vulnerable a la violencia en la calle o en los albergues. En cuanto a violencia intrafamiliar se da un patrón similar (Ver Figura 8). Las mujeres que están en la actualidad sin hogar tienen mayor probabilidad de informar haber sido víctimas de violencia intrafamiliar $(\mathrm{p}<, 05)$. Tales experiencias pueden afectar sus opciones de vivienda y generarles temor respecto a los albergues u opciones de viviendas colectivas. Muchas de las mujeres que informaron contar con vivienda están habitando lugares inseguros y/o inestables. Viven con parientes, amigos y "parejas", quienes podrían obligarlas a dejar el hogar sin previo aviso. Debido a sus precarias finanzas, las mujeres y sus seres queridos pueden estar imposibilitados de pagar el arriendo, lo que resulta en la carencia de hogar. Algunas de las mujeres que viven en albergues informan tener hogar si bien otras se identificaron como carentes de éste. Las opciones de albergues temporales incluyen albergues de corto y largo plazo, así como programas de hogares de transición. Es preocupante el hecho de que las mujeres que habían experimentado abuso sexual y/o violencia intrafamiliar tuvieran mayor probabilidad de estar sin hogar. La carencia de hogar hace a las mujeres más vulnerables respecto de la violencia y el abuso sexual, y más proclives a involucrarse en el trabajo sexual con fines de subsistencia.

RELACIONES SIGNIFICATIVAS ENTRE LOS FACTORES DE RIESGO ASOCIADOS AL ESTATUS DE VIVIENDA EN LA MUESTRA DE MUJERES

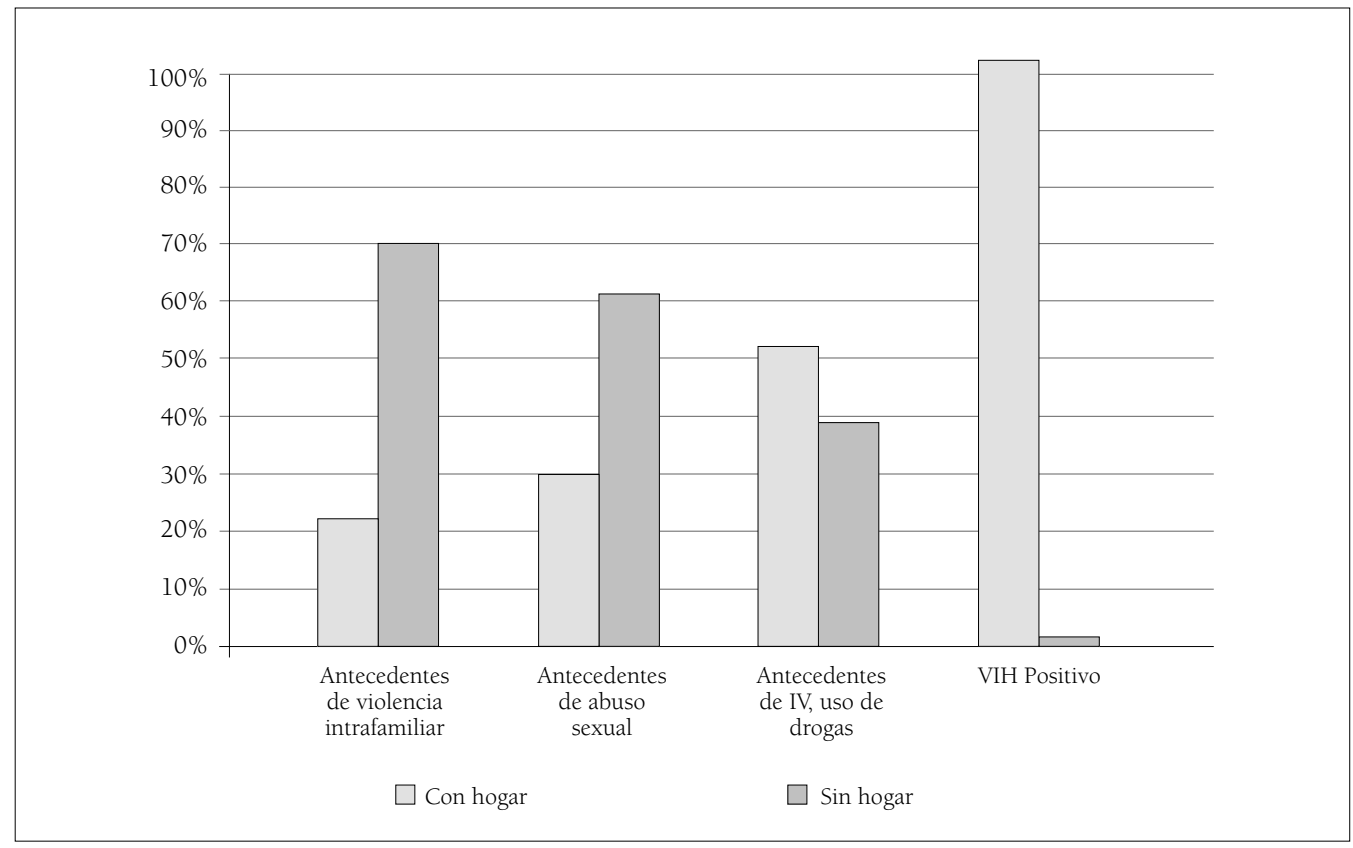




\section{Conclusiones sobre género y carencia de hogar}

Una serie de análisis con tabulación cruzada en tres sentidos intentó encontrar la relación entre ambos géneros y la carencia de hogar con respecto a los factores incluidos en este estudio. Se compararon cuatro grupos: hombres sin hogar, mujeres sin hogar, hombres con hogar, mujeres con hogar.

Hubo diversas conclusiones importantes en relación a las mujeres en estos análisis. Las mujeres con VIH positivo tuvieron mayor probabilidad de contar con hogar ( $\mathrm{p}<, 05)$. Las mujeres sin hogar tuvieron menor probabilidad de involucrarse en trabajo sexual con respecto a aquellas que sí tenían un lugar donde vivir. $(\mathrm{p}=, 025)$. Las mujeres sin hogar habían experimentado más abuso sexual que aquéllas que si tenían hogar $(\mathrm{p}<, 05)$. Casi el doble de las mujeres sin hogar experimentó violencia intrafamiliar $(p<, 05)$. Las mujeres sin hogar mostraron tasas inferiores de uso de drogas inyectables $(p=, 004)$ y tuvieron mayor probabilidad de informar no haber utilizado nunca crack en comparación con las mujeres con hogar $(\mathrm{p}=, 002)$. Ver Figura 9.

Hubo una importante conclusión en relación a los hombres sin hogar y las mujeres con hogar. Un mayor porcentaje de hombres sin hogar tenía parejas cuyo uso de drogas inyectables fue reconocido ( $\mathrm{p}<, 05)$.

En dos de los análisis, hubo conclusiones importan- tes tanto para los hombres como para las mujeres. Las mujeres sin hogar mostraron tasas menores de uso de drogas inyectables que las mujeres con hogar $(p=, 004)$. Los hombres sin hogar revelaron tasas más elevadas de uso de drogas inyectables con respecto a los hombres con hogar $(p<, 05)$. En el uso del crack se descubrió un patrón similar. Un porcentaje mayor de mujeres sin hogar respecto de las que sí tenían hogar nunca había usado crack $(p=, 002)$, en tanto los hombres sin hogar tuvieron más probabilidad que aquellos que sí tenían hogar de usar crack $(\mathrm{p}<, 05)$.

Estas conclusiones confirman aquellas conclusiones de la sección anterior de este análisis en cuanto a género y carencia de hogar. Demuestran la compleja matriz de factores que no pueden separarse fácilmente para comprender los factores de riesgo de un individuo. La capacidad de mantener una vivienda podría estar relacionada con factores económicos y con el acceso a programas de vivienda. Las mujeres involucradas en trabajo sexual pueden permitirse pagar por una vivienda, aún cuando particulares poblaciones (de personas que abusan de sustancias, o de aquellas con VIH positivo) pueden tener mayores opciones de vivienda que los demás. Y aún cuando cuenten con un lugar donde vivir, las poblaciones marginadas pueden estar utilizando albergues $u$ otras organizaciones de vivienda inadecuadas o inestables, como por ejemplo habitar en un "hogar del crack" e intercambiar sexo por drogas u hospedaje.

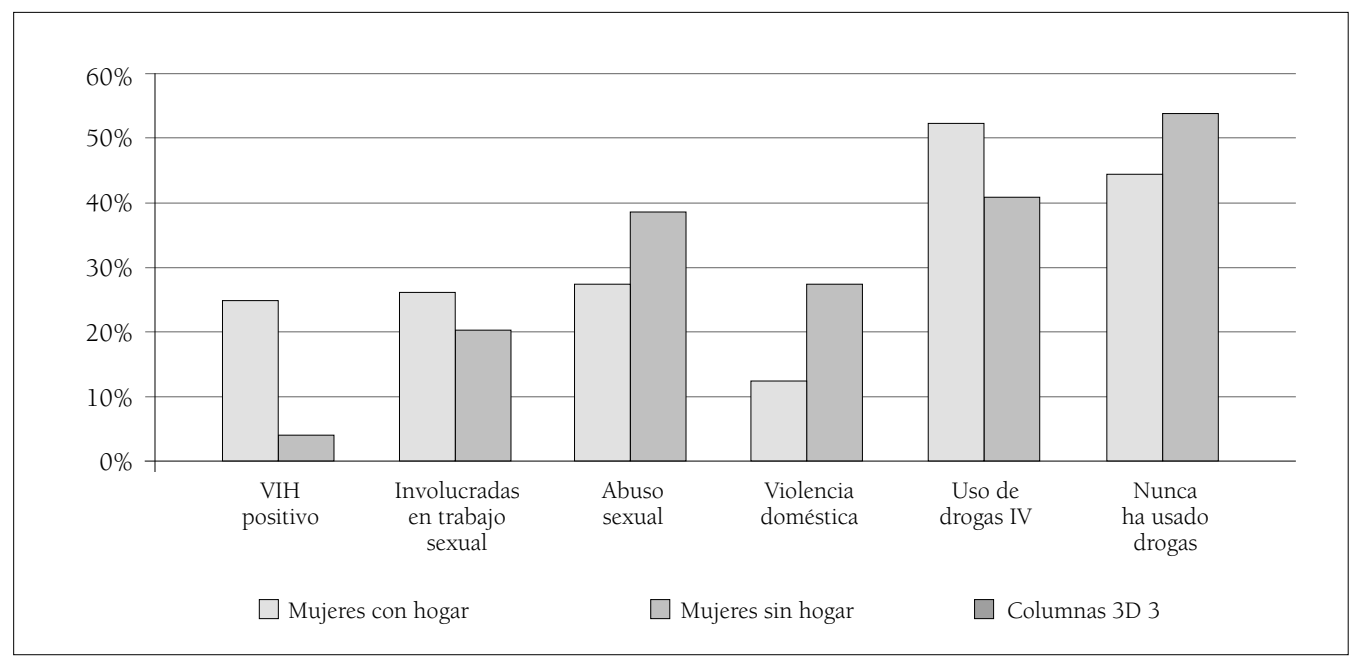




\section{Consecuencias en el trabajo y en la práctica del Trabajo Social}

Este artículo describe los hallazgos relevantes para la comprensión de la naturaleza compleja de la vida en las calles y para el desarrollo de contenidos de un curso que ayude a los estudiantes de trabajo social a comprender los factores que afectan a las personas sin hogar y que a su vez son factores afectados por la situación de calle. Este estudio demuestra la compleja matriz de factores interrelacionados que generan vulnerabilidad, incluyendo el género, carencia de hogar, uso de drogas, violencia y VIH. Los hallazgos individuales destacan los problemas de justicia social que son inherentes a las diferencias de género entre las personas sin hogar, ofreciendo ejemplos concretos con los cuales se puede ayudar a los estudiantes a entender las inequidades que afectan las vidas de las personas y las alternativas que tienen a su disposición.

Específicamente en cuanto a las mujeres, las inequidades incluyen el rol que juegan el abuso sexual y la violencia intrafamiliar en el consiguiente trabajo sexual, uso de drogas y carencia de hogar. Las realidades biológicas del VIH hacen a las mujeres sin hogar más vulnerables, por sus encuentro sexuales con hombres. Esta población de mujeres no cuenta con acceso fácil a los servicios sociales y necesita ser identificada por servicios de bajo umbral, que les impongan pocos requerimientos para poder postular a sus servicios. Esto queda evidenciado por el bajo nivel de participación en los programas médicos asistenciales (7\%) de esta gran muestra, habiendo porcentajes mucho mayores que demostraron necesidades de atención médica que justificaban su calificación para recibir asistencia médica pública. Estas mujeres en riesgo requieren servicios básicos muy concretos, tales como hospedaje, cuidados médicos, servicios de protección y desintoxicación. Estos servicios necesitan entregarse en forma sensible en lo cultural y de manera que no aumenten su stress y riesgo.

Estos análisis de datos ofrecen la oportunidad de discutir los factores que se relacionan con las poblaciones marginadas y que pueden utilizarse para ilustrar los problemas de género, carencia de hogar, uso de drogas, trabajo sexual y violencia mediante los cursos de práctica del trabajo social, de comportamiento humano en el entorno social (CHCS) y políticas. Muchas de estas conclusiones están abiertas a múltiples interpretaciones y les permiten a los estudiantes el pensar acerca de problemas que no son tan simples o que no se evidencian en lo inmediato.

\section{Bibliografía}

BARROW, S.M., HERMAN, D.P., CORDOVA, P., \& STREUNING, E.L. (1999). Mortality among homeless shelter residents in New York City. American Journal of Public Health, 89(4), 529-534.

BLAKENSHIP, K.M. \& KOESTER, S. (2002). Criminal law, policing policy and HIV risk in female street sex workers and injection drug users. Journal of Law, Medicine and Ethics, 30, 548-559.

CHEUNG, A.M., \& HWANG, S.H. (2004). Risk of death among homeless women: A cohort study and review of the literature. Canadian Medical Association Journal, 170 (8), 1243-1247

CHRISTENSEN, R.C., HODGKINS, C.C., GARCES, L.K., ESTLUND, K.L., MILLER, M.D., \& TOUCHTON, R. (2005). Homeless, mentally ill and addicted: The need for abuse and trauma services. Journal of Health Care for the Poor and Underserved, 16 (4), 615-621.

ERICKSON, P.G., BUTTERS, J., MCGILLICUDDY, P., \& HALLGREN, A. (2000). Crack and prostitution: Gender, myths and experiences. Journal of Drug Issues, 30 (4), 767-788.

HARVEY, S.M., BIRD, S.T., DE ROSA, C.J., MONTGOMERY, S.B., \& ROHRBACH, L.A. (2003). Sexual decision making and safer sex behavior among young female injection drug users and female partners of IDUs. The Journal of Sex Research, 40 (1), 50-60.

LEWIS, J.H., ANDERSEN, R.M., \& GELBERG, L. (2003). Health care for homeless women: Unmet needs and barriers to care. Journal of General Internal Medicine, 18 (11), 921-928.

MULIA, N. (2000). Questioning sex: Drug using women and heterosexual relations. Journal of Drug Issues, 30 (4), 741-766.

SURRATT, H.L., \& INCIARDI, J.A. (2004). HIV risk, seropositivity and predictors of infection among homeless and non-homeless women sex workers in Miami, Florida, USA. AIDS Care, 16 (5), 594-604

WEINER, A. (1996). Understanding the social needs of streetwalking prostitutes. Social Work, 41 (1), 97-105.

WITTE, S.S., EL-BASSEL, N., WADA, T., GRAY, O., \& WALLACE, J. (1999). Acceptability of female condom use among women exchanging street sex in New York City. International Journal of STDS \& AIDS, 10, 162-168.

ZUGAZAGA, C. (2004). Stressful life event experiences of homeless adults: A comparison of single men, single women and women with children. Journal of Community Psychology, 32 (6), 643-654. 